\title{
A STUDY OF ALMOST-EVERYWHERE SINGLETON-VALUED FILIPPOVS
}

\author{
DANIEL C. BILES AND JOHN S. SPRAKER
}

(Communicated by Kenneth R. Meyer)

\begin{abstract}
Filippov solutions of $x^{\prime}(t)=f(t, x(t))$ are defined in terms of a certain set-valued function called the Filippov of $f$. Under some fairly general assumptions, we answer the following question: The Filippovs of $f$ and $g$ are equal if and only if $f$ and $g$ relate in what way?
\end{abstract}

\section{INTRODUCTION}

Let $f: \mathbf{R} \rightarrow \mathbf{R}$ be given. We define the Filippov of $f$ as follows:

$$
\mathscr{F}[f](x)=\bigcap_{\varepsilon>0} \bigcap_{Z: m(Z)=0} \overline{\operatorname{conv}} f\left(B_{\varepsilon}(x) \backslash Z\right),
$$

where $m$ denotes Lebesgue measure, $\overline{\operatorname{conv}} A$ represents the closure of the convex hull of the set $A$ and $B_{\varepsilon}(x)$ represents the open ball of radius $\varepsilon$ about the point $x$. Note that the Filippov is a set-valued function. The Filippov is used in defining a generalized solution of the ordinary differential equation $x^{\prime}=f(t, x)$, particularly in the case of $f$ discontinuous in $x$ (see, for example $[1,2,3])$.

In this paper we investigate the following question:

$$
\mathscr{F}[f]=\mathscr{F}[g] \text { if and only if } f \text { and } g \text { relate in what way? }
$$

(It is not difficult to show that if $f(x)=g(x)$ a.e., then $\mathscr{F}[f]=\mathscr{F}[g]$, since the Filippov operator "ignores" sets of measure zero.) To this end, consider the following. It is known that there exists a Lebesgue measurable set $A \subseteq \mathbf{R}$ such that for all $x \in \mathbf{R}$ and $\varepsilon>0$, we have $0<m\left(A \cap B_{\varepsilon}(x)\right) / m\left(B_{\varepsilon}(x)\right)<1$ (see, for example, [4]). Define $f, g: \mathbf{R} \rightarrow \mathbf{R}$ by $f(x)=\mathscr{X}_{A}(x)$ and $g(x)=\mathscr{X}_{A^{c}}(x)$ where $\mathscr{X}_{A}(x)$ and $\mathscr{X}_{A c}(x)$ denote the characteristic functions of $A$ and $\mathbf{R} \backslash A$ respectively. It can be shown that for each $x \in \mathbf{R}, \mathscr{F}[f](x)=\mathscr{F}[g](x)=[0,1]$. However, $f$ and $g$ do not agree anywhere. Thus, it appears that, in general, there is no "nice" characterization of the relationship between $f$ and $g$ in the case in which $\mathscr{F}[f]=\mathscr{F}[g]$ (without repeating the definition in some trivial way). However, we show in this paper that under fairly general assumptions, a

Received by the editors August 4, 1990.

1980 Mathematics Subject Classification (1985 Revision). Primary 34A10; Secondary 34A34.

Key words and phrases. Filippov solution. 
"nice" characterization does exist. Specifically, we obtain a characterization of the relationship between $f$ and $g$ in the case in which their Filippovs are singletons almost everywhere. We also give necessary and sufficient conditions for a function $f$ to have a Filippov that satisfies this condition, plus necessary and sufficient conditions for $f$ to have a Filippov with other properties of interest. In $\S 2$ we present some technical lemmas. In $\S 3$ we present and prove our main results.

\section{Technical Lemmas}

This section consists of a list of lemmas and their proofs, which are used to obtain the main results in the final section. We assume throughout that $f, g: \mathbf{R} \rightarrow \mathbf{R}$ are Lebesgue measurable.

Lemma 1. Let $f: \mathbf{R} \rightarrow \mathbf{R}$ be given. If there exists a set $A \subseteq \mathbf{R}$ of full measure such that $\left.f\right|_{A}$ is continuous, then for each $x \in A$ we have $\mathscr{F}[f](x)=\{f(x)\}$.

Proof.

Claim. For each $x \in A$ we have $f(x) \in \mathscr{F}[f](x)$.

Proof of Claim. Fix $\varepsilon>0$ and $Z \subseteq \mathbf{R}$ with $m(Z)=0$. Since $A$ has full measure, then for each $n \in \mathbf{N}, A \cap\left(B_{1 / n}(x) \backslash Z\right)$ has full measure in $B_{1 / n}(x)$. Thus for any $n$, there exists $x_{n} \in A \cap\left(B_{1 / n}(x) \backslash Z\right)$. Clearly $x_{n} \rightarrow x$ and the continuity of $f$ relative to $A$ yields that $f\left(x_{n}\right) \rightarrow f(x)$. Thus $f(x) \in \operatorname{cl} f\left(B_{\varepsilon}(x) \backslash Z\right) \subseteq$ $\overline{\text { conv }} f\left(B_{\varepsilon}(x) \backslash Z\right)$. Since the above statements hold for any $\varepsilon>0$ and $Z \subseteq \mathbf{R}$ of measure zero, we have $f(x) \in \bigcap_{\varepsilon>0} \bigcap_{Z: m(Z)=0} \overline{\operatorname{conv}} f\left(B_{\varepsilon}(x) \backslash Z\right)=\mathscr{F}[f](x)$ so the claim is established.

Assume there exists an $\bar{x} \in A$ such that $\mathscr{F}[f](\bar{x}) \neq\{f(\bar{x})\}$. Thus, there exists $y \in \mathbf{R}$ such that $y \neq f(\bar{x})$ and $y \in \mathscr{F}[f](\bar{x})$. (Note: we know $\mathscr{F}[f](\bar{x})$ is nonempty because of the claim.) Without loss of generality, assume that $y>f(\bar{x})$. For each $\varepsilon>0$ and $Z \subseteq \mathbf{R}$ with $m(Z)=0$, we have that $y \in$ $\overline{\text { conv }} f\left(B_{\varepsilon}(\bar{x}) \backslash Z\right)$. Let $Z_{0}=\mathbf{R} \backslash A$. Then for each $\varepsilon>0, y \in \overline{\operatorname{conv}} f\left(B_{\varepsilon}(\bar{x}) \backslash Z_{0}\right)$. Thus, for every $\varepsilon>0$ there exist $l_{\varepsilon}$ and $u_{\varepsilon}$ such that $l_{\varepsilon} \leq y \leq u_{\varepsilon}$ and $l_{\varepsilon}, u_{\varepsilon} \in f\left(B_{\varepsilon}(\bar{x}) \backslash Z_{0}\right)$. For each $\varepsilon>0$ let $w_{\varepsilon} \in B_{\varepsilon}(\bar{x}) \backslash Z_{0}=B_{\varepsilon}(\bar{x}) \cap A$ satisfy $f\left(w_{\varepsilon}\right)=u_{\varepsilon}$. Let $\beta=y-f(\bar{x})$. We have thus shown that there exists $\beta>0$ such that for each $\varepsilon>0$ there exists $w_{\varepsilon} \in B_{\varepsilon}(\bar{x}) \cap A$ with $f\left(w_{\varepsilon}\right)-f(\bar{x})=$ $u_{\varepsilon}-f(\bar{x}) \geq y-f(\bar{x})=\beta$. This contradicts the continuity of $\left.f\right|_{A}$ at $\bar{x}$.

Lemma 2. Let $f: \mathbf{R} \rightarrow \mathbf{R}$ be given. Then $\mathscr{F}[f]$ is continuous when restricted to the set $A$ on which it is a singleton (treated as a real-valued function).

Proof. Assume not. Then there exists some $\bar{x} \in A$ at which $\mathscr{F}[f]$ is discontinuous. Thus, there exists $\beta>0$ such that at least one of the following holds:

1. For every $\varepsilon>0$, there exists $y_{\varepsilon} \in A$ with $\left|\bar{x}-y_{\varepsilon}\right|<\varepsilon$ but $\mathscr{F}[f]\left(y_{\varepsilon}\right)-$ $\mathscr{F}[f](\bar{x}) \geq \beta$.

2. For every $\varepsilon>0$, there exists $y_{\varepsilon} \in A$ with $\left|\bar{x}-y_{\varepsilon}\right|<\varepsilon$ but $\mathscr{F}[f]\left(y_{\varepsilon}\right)-$ $\mathscr{F}[f](\bar{x}) \leq-\beta$.

Without loss of generality, assume that 1 holds. Choose $\varepsilon>0$ and take a corresponding $y_{\varepsilon} \in A$. We know for every $\gamma>0$ and every $Z \subseteq \mathbf{R}$ with $m(Z)=$ 0 , that $\mathscr{F}[f]\left(y_{\varepsilon}\right) \in \overline{\operatorname{conv}} f\left(B_{\gamma}\left(y_{\varepsilon}\right) \backslash Z\right)$. Choose $\gamma>0$ so that $B_{\gamma}\left(y_{\varepsilon}\right) \subseteq B_{\varepsilon}(\bar{x})$. We then have for each $Z \subseteq \mathbf{R}$ of measure zero that $B_{\gamma}\left(y_{\varepsilon}\right) \backslash Z \subseteq B_{\varepsilon}(\bar{x}) \backslash Z$ and 
so $f\left(B_{\gamma}\left(y_{\varepsilon}\right) \backslash Z\right) \subseteq f\left(B_{\varepsilon}(\bar{x}) \backslash Z\right)$. Thus we have $\mathscr{F}[f]\left(y_{\varepsilon}\right) \in \overline{\text { conv }} f\left(B_{\gamma}\left(y_{\varepsilon}\right) \backslash Z\right) \subseteq$ $\overline{\text { conv }} f\left(B_{\varepsilon}(\bar{x}) \backslash Z\right)$. That is, we have shown that for every $\varepsilon>0$ and $Z \subseteq \mathbf{R}$ with $m(Z)=0, \overline{\operatorname{conv}} f\left(B_{\varepsilon}(\bar{x}) \backslash Z\right) \cap\{y: y \geq \beta+\mathscr{F}[f](\bar{x})\} \neq \varnothing$. Thus for every $\varepsilon>0$ and every $Z \subseteq \mathbf{R}$ with $m(Z)=0$, we have $[\mathscr{F}[f](\bar{x}), \mathscr{F}[f](\bar{x})+\beta] \subseteq$ $\overline{\text { conv }} f\left(B_{\varepsilon}(\bar{x}) \backslash Z\right)$, and thus

$$
[\mathscr{F}[f](\bar{x}), \mathscr{F}[f](\bar{x})+\beta] \subseteq \bigcap_{\varepsilon>0} \bigcap_{Z: m(Z)=0} \overline{\operatorname{conv}} f\left(B_{\varepsilon}(\bar{x}) \backslash Z\right)=\mathscr{F}[f](\bar{x}),
$$

which is a contradiction.

Lemma 3. Let $f: \mathbf{R} \rightarrow \mathbf{R}$ be given. If $\mathscr{F}[f]$ is a singleton almost everywhere, then $\mathscr{F}[f](x)=\{f(x)\}$ almost everywhere.

Proof. Assume the opposite. Then without loss of generality, it follows that there exists an $A \subseteq \mathbf{R}$ such that $m(A)>0, \mathscr{F}[f](x)$ is a singleton for every $x \in A$ and $\mathscr{F}[f](x)<f(x)$ for every $x \in A$.

Claim 1. There exists $\delta>0$ such that $m\{x \in A \mid f(x)>\mathscr{F}[f](x)+\delta\}>0$.

Proof of Claim 1. Assume the opposite. Then for $\delta=1 / n, n=1,2, \ldots$, $m\{x \in A \mid f(x)>\mathscr{F}[f](x)+1 / n\}=0$. But

$$
A=\bigcup_{n=1}^{\infty}\{x \in A \mid f(x)>\mathscr{F}(f)(x)+1 / n\},
$$

a countable union of sets of measure zero, which implies $m(A)=0$, a contradiction.

Now let $y$ be a point of density of $B \equiv\{x \in A \mid f(x)>\mathscr{F}[f](x)+\delta\}$ such that $y \in B$.

Claim 2. There exists $\bar{\varepsilon}>0$ such that

$$
\inf _{x \in B_{\bar{\varepsilon}}(y) \cap A}\{\mathscr{F}[f](x)+\delta-\mathscr{F}[f](y)\}>0 .
$$

Proof of Claim 2. Define $g: C \rightarrow \mathbf{R}$ by $g(x)=\mathscr{F}[f](x)+\delta-\mathscr{F}[f](y)$ where $C$ is the set on which $\mathscr{F}[f]$ is a singleton. By Lemma $2, \mathscr{F}[f]$ is continuous restricted to the set $C$, hence $g$ is continuous. The fact that $g(y)=\delta>0$ and the continuity of $g$ restricted to $C$ implies that there exists $\bar{\varepsilon}>0$ such that $\inf _{x \in B_{\bar{\varepsilon}}(y) \cap C}\{\mathscr{F}[f](x)+\delta-\mathscr{F}[f](y)\}>0$. Noting that $A \subseteq C$, the claim follows.

Because $y$ is a point of density of $B$, we know that for all $\varepsilon>0$,

$$
m\left[B_{\varepsilon}(y) \cap\{x \in A \mid f(x)>\mathscr{F}[f](x)+\delta\}\right]>0 .
$$

Let

$$
p=\inf _{x \in B_{\bar{\varepsilon}}(y) \cap A}\{\mathscr{F}[f](x)+\delta-\mathscr{F}[f](y)\} .
$$

Claim 3. For all $\varepsilon>0, m\left[B_{\varepsilon}(y) \cap\{x \in A \mid f(x)>\mathscr{F}[f](y)+p\}\right]>0$.

Proof of Claim 3. Suppose the opposite. Then, there exists $\hat{\varepsilon}>0$ such that $m\left[B_{\hat{\varepsilon}}(y) \cap\{x \in A \mid f(x)>\mathscr{F}[f](y)+p\}\right]=0$. Choose $\varepsilon=\min \{\hat{\varepsilon}, \bar{\varepsilon}\}$ (where $\bar{\varepsilon}$ is chosen as in Claim 2). Then, $B_{\hat{\varepsilon}}(y) \cap\{x \in A \mid f(x)>\mathscr{F}[f](y)+p\} \supseteq$ $B_{\varepsilon}(y) \cap\{x \in A \mid f(x)>\mathscr{F}[f](y)+p\} \supseteq B_{\varepsilon}(y) \cap\{x \in A \mid f(x)>\mathscr{F}[f](x)+\delta\}$ 
by the previous claim. This last set has positive measure (by Claim 1), which yields a contradiction.

Thus for each $\varepsilon>0$ and for every $Z \subseteq \mathbf{R}$ with $m(Z)=0$, we have $\overline{\operatorname{conv}} f\left(B_{\varepsilon}(y) \backslash Z\right) \supseteq[\mathscr{F}[f](y), \mathscr{F}[f](y)+p]$ and thus $[\mathscr{F}[f](y), \mathscr{F}[f](y)+p] \subseteq$ $\mathscr{F}[f](y)$, which is a contradiction.

\section{THE MAIN RESULTS}

We now present and prove the main results of this paper. We first present theorems that characterize $f$, depending on the properties of its Filippov. The first result characterizes $f$ in the case in which it is its own Filippov.

Theorem 1. Let $f: \mathbf{R} \rightarrow \mathbf{R}$ be given. For each $x \in \mathbf{R}, \mathscr{F}[f](x)=\{f(x)\}$ if and only if $f$ is continuous.

Proof. $(\Rightarrow)$ : By Lemma 2, $\mathscr{F}[f]$ is continuous. Thus $f$, being the same function, has the same property.

$(\Leftarrow)$ : This follows from Lemma 1 , letting $A=\mathbf{R}$.

The following result characterizes $f$ in the case in which its Filippov is always a singleton (not necessarily $\{f\}$ ).

Theorem 2. Let $f: \mathbf{R} \rightarrow \mathbf{R}$ be given. For each $x \in \mathbf{R}, \mathscr{F}[f](x)$ is a singleton if and only if $f$ agrees a.e. with a continuous function $g: \mathbf{R} \rightarrow \mathbf{R}$.

Proof. $(\Rightarrow)$ : We need to construct a $g: \mathbf{R} \rightarrow \mathbf{R}$ with two properties:

(a) $g$ is a continuous, and

(b) $f=g$ almost everywhere.

Define $g$ to be the function that satisfies $\mathscr{F}[f](x)=\{g(x)\}$ for each $x \in \mathbf{R}$. The (a) requirement follows from Lemma 2 , since $\mathscr{F}[f](x)$ is a singleton for each $x \in \mathbf{R}$. The (b) requirement follows from Lemma 3. It tells us that $\mathscr{F}[f](x)=\{f(x)\}$ a.e. and thus $f(x)=g(x)$ a.e.

$(\Leftarrow)$ : Let $g: \mathbf{R} \rightarrow \mathbf{R}$ represent a function that is continuous and satisfies $g(x)=f(x)$ a.e. It follows easily that $\mathscr{F}[g](x)=\mathscr{F}[f](x)$ for all $x \in \mathbf{R}$. By Lemma $1, \mathscr{F}[g](x)=\{g(x)\}$ for all $x \in \mathbf{R}$. Thus, for any $x \in \mathbf{R}$, $\mathscr{F}[f](x)=\mathscr{F}[g](x)=\{g(x)\}$, a singleton.

We now characterize $f$ in the case in which its Filippov is a singleton almost everywhere. As a "bonus," we get the equivalence of these two conditions with a third property, which is condition (ii) mentioned below.

Theorem 3. Let $f: \mathbf{R} \rightarrow \mathbf{R}$ be given. The following three properties are equivalent:

(i) $\mathscr{F}[f](x)$ is a singleton almost everywhere.

(ii) $\mathscr{F}[f](x)=\{f(x)\}$ almost everywhere.

(iii) There exists a set $A \subseteq \mathbf{R}$ of full measure such that $\left.f\right|_{A}$ is continuous.

Proof. (i) $\Rightarrow$ (ii): This is precisely Lemma 3 .

(ii) $\Rightarrow($ iii): $\mathscr{F}[f](x)$ is a singleton a.e., hence, by Lemma $2, \mathscr{F}[f]$ is continuous when restricted to a set of full measure, call it $S_{1}$. By hypothesis, $\mathscr{F}[f](x)=\{f(x)\}$ on a set of full measure, call it $S_{2}$. We now let $A=S_{1} \cap S_{2}$, and note that $\left.f\right|_{A}$ is continuous.

(iii) $\Rightarrow$ (ii): Follows easily from Lemma 1 . 
(ii) $\Rightarrow$ (i): Trivial, since, for each $x \in \mathbf{R},\{f(x)\}$ is a singleton.

Remark. Clearly, the properties listed in Theorem 2 imply those of Theorem 3. However, the converse is not true, since it is easy to construct an $f$ with a Filippov, which is a singleton only almost everywhere, say, $f: \mathbf{R} \rightarrow \mathbf{R}$ given by $f(x)=1$ for $x<0$ and $f(x)=-1$ for $x \geq 0$.

We now consider the question posed in the introduction: $\mathscr{F}[f]=\mathscr{F}[g]$ if and only if $f$ and $g$ relate in what way? The following theorem covers the case in which the Filippovs are singletons almost everywhere. Note also that we include a third property, which tells us that in the aforementioned case, it is impossible for the Filippovs to agree on a set of full measure, but disagree on a set of zero measure (except, of course, for the null set).

Theorem 4. Let $f, g: \mathbf{R} \rightarrow \mathbf{R}$ be given such that both $\mathscr{F}[f](x)$ and $\mathscr{F}[g](x)$ are singletons a.e. Then, the following three properties are equivalent:

(i) For each $x \in \mathbf{R}, \mathscr{F}[f](x)=\mathscr{F}[g](x)$.

(ii) $\mathscr{F}[f](x)=\mathscr{F}[g](x)$ almost everywhere.

(iii) $f(x)=g(x)$ almost everywhere.

Proof. (i) $\Rightarrow$ (ii): Trivial.

(ii) $\Rightarrow$ (iii): Since $\mathscr{F}[f](x)$ is a singleton a.e., we have by Lemma 3 that $\mathscr{F}[f](x)=\{f(x)\}$ a.e. Similarly, $\mathscr{F}[g](x)=\{g(x)\}$ a.e. Thus, by hypothesis, $\{f(x)\}=\{g(x)\}$ a.e., that is $f(x)=g(x)$ a.e.

(iii) $\Rightarrow$ (i): Clear, since the construction of the Filippov "ignores" sets of measure zero.

\section{REFERENCES}

1. A. F. Filippov, Differential equations with discontinuous right-hand side, Mat. Sb. 51 (1960), 99-128 (Russian); Amer. Math. Soc. Transl. Ser. 2, vol. 42, Amer. Math. Soc., Providence, RI, 1964, pp. 199-231.

2. __ Differential equations with discontinuous right-hand sides, Kluwer, Boston, 1988.

3. O. Hájek, Discontinuous differential equations, I, J. Differential Equations 32 (1979), 149170.

4. A. Simoson, An "Archimedean" paradox, Amer. Math. Monthly, vol. 89, no. 2, Math. Assoc. Amer., Washington, DC, 1982, pp. 114-125.

Department of Mathematics, Western Kentucky University, Bowling Green, KENTUCKY 42101 